\title{
Monitoring of Breast Lesions after Breast Conservative Therapy: Does A Combination of Dynamic and Functional MRI Techniques Can Add Value in Early Detection of Recurrent Malignancy?
}

\author{
MOHAMAD F. SHERIF, M.D.*; NAGLA L. DABEES, M.D.*; SAMAR G. YONES, M.D.** and \\ OM YOSEF A. KHEDR, M.D.**,*** \\ The Departments of Diagnostic Radiology* and Oncology \& Nuclear Medicine**, Faculty of Medicine, Tanta University and \\ The Department of Medicine, Faculty of Medicine, Al-Menshawy Hospital***, Tanta
}

\begin{abstract}
Background: To assess efficacy of combined dynamic and functional MRI techniques in early detection of recurrent malignancy after breast conservative therapy and differentiating it from other post-operative lesions.

Aim of Study: To assess efficacy of combined dynamic and functional MRI techniques in early detection of recurrent malignancy after breast conservative therapy and differentiating it from other post-operative lesions.

Patients and Methods: Thirty female patients who underwent breast conservative therapy were involved in this prospective study. Patients were examined by sono-mammography that aroused suspicious of breast lesions followed by DCEMRI \& DWI-MRI to exclude recurrence. DWI was performed using b-values of $50,400,800 \mathrm{~s} / \mathrm{mm}^{2}$. All patients were subjected to histopathological confirmation which was the gold standard in this study.

Results: Eight patients had pathologically proven recurrence and 22 patients showed spectrum of post-operative changes. DCE- MRI diagnosis (considering lesion morphology, enhancement pattern) overestimated 2 lesions were scored BIRADS 4 and proved pathologically as fat necrosis.
\end{abstract}

When adding the data elicited by the DWI to the DCEMRI assessment, the statistical analysis presented $100 \%$ sensitivity, 95.4\% specificity, $88.9 \%$ PPV, 100\% NPV and $96.6 \%$ accuracy.

Conclusion: Combination of functional \& dynamic MRI techniques added high significant value in follow-up of breast lesion occurred after BCT as it can avoid biopsy in positive patients, to send negative patients to follow-up and reserving biopsy in the cases with a clinico-radiological mismatch. Breast MRI should be the imaging modality of choice in detection of the tumoral recurrence, and differentiating it from other post-operative lesions.

Key Words: Breast Conservative Therapy - Dynamic and Functional MRI Techniques - Early Detection of Recurrent Malignancy.

Correspondence to: Dr. Mohamad F. Sherif, The Department of Diagnostic Radiology, Faculty of Medicine,

Tanta University, Tanta

\section{Introduction}

THE breast cancer is the commonest cancer in female, representing $27 \%$ of all female malignancy [1]. Breast-Conserving Therapy (BCT) is progressively being included into treatment of breast cancer, it is either lumpectomy, lumpectomy with dissection of axillary lymph nodes or quadrectomy with dissection of axillary lymph nodes followed by breast radiotherapy, these being the standard of management for early-stage breast cancer (stages I and II) [2] . After BCT, the occurrence of local recurrence is ranged between 1:2\% per year [3] Post-operative scar may cause significant clinical problems if the recurrent malignancy is suspected, as it exhibited severe architectural distortion, with focal increases breast density on mammogram or even seems as breast mass [4,5]. Magnetic Resonance Imaging (MRI) can detect multifocality of breast cancer, differentiating scar tissue from recurrent cancer following BCT [6]. Dynamic Contrast-Enhanced Magnetic Resonance Imaging (DCE-MRI) has important role in patients treated with BCT. Diffusion Weighted Imaging (DWI) assess random free water molecules motion and characterizes different tissues [7,8]. DCE-MRI and

\footnotetext{
Abbreviations :

BCT : Breast Conservative Therapy.

MRI : Magnetic Resonance Imaging.

DCE : Dynamic Contrast Enhancement.

DWI : Diffusion Weighted Images.

BIRADS : Breast Imaging-Reporting and Data System.

S.I. : Signal Intensity.

ROI : Region of Interest.

ADC : Apparent Diffusion Coefficient.

SD : Standard Deviation.

ROC : Receiver Operating Characteristic.
} 
DWI-MRI have improving early detection of tumor response to treatment and post therapeutic evaluation of residual tumor $[\mathbf{9 , 1 0 ]}$

\section{Patients and Methods}

\section{Patients:}

This prospective study was carried out on 30 female patients who underwent BCT for breast cancer with suspicious findings in ultrasound and/or mammography on follow-up. In our study 4 patients $(13.3 \%)$ were $<40$ years old and 26 patients $(86.7 \%)$ were $>40$ years old. The study was performed at period from April 2017 to March 2019.

\section{Inclusion criteria:}

Patients underwent BCT for breast cancer with suspected local recurrence detected at mammography, ultrasound and/or clinical examination during routine follow-up. Patients with newly developed lesions at their operative bed. Lesions detected on sono-mammographic examinations that were scored according to Breast Imaging-Reporting and Data System (BIRADS) from BIRADS 3 to BIRADS 5.

\section{Exclusion criteria:}

Patients with post-operative lesions that scored BIRADS 2 at sono-mammographic examination. Contraindications to gadolinium-based contrast media: Pregnant women, chronic renal impairment (serum creatinine $>1.5 \mathrm{mg} / \mathrm{dl}$ ), previous allergy to the contrast media, clinically significant cardiac arrhythmias or congestive heart failure. Inability to lie prone, marked obesity, extremely large breasts, implantable devices that are not MRI compatible.

The study's protocol was approved by Research Ethics Committee and Quality Assurance Unit. The patients were aware of examination, informed consent was obtained from patients.

\section{All patients were subjected to full history taking:}

I- Personal, menstrual, breast oncologic, family and present histories.

II- Meticulous clinical examination of the mass.

\section{Methods:}

I- Sono-mammographic examinations were done for all patients as screening test.

II- Magnetic Resonance Imaging (MRI): MRI studies were done for 30 patients using a closed MRI machine (General Electric SIGNA-high speed) with magnets of intensity field $1.5 \mathrm{~T}$. Patients were placed on top of dedicated specialized phased array breast coils in prone position with breast support by pads and cushions. Premenopausal women underwent the examination on day 6-13 of the menstrual cycle to reduce risk of false positive results. Time interval between surgery, recent/ ongoing radiotherapy sessions and contrast enhanced MRI examination was 6-18 months after surgery to avoid operative bed enhancement. The patients were examined by:

A- Fast spin echo T1WI, T2WI with fat suppression in transverse plane; slice thickness: $4 \mathrm{~mm}$; spacing: $1 \mathrm{~mm}$; image matrix: 320 X 314; 4 NEX.

B- DWI of the whole breast in transverse plane with a single excitation echo planar imaging sequence. A diffusion-sensitive gradient was performed at X-, Y-, and Z-axes. Parameters of DWI were: TR $8400 \mathrm{~ms}$, TE $98 \mathrm{~ms}$; slice thickness $4 \mathrm{~mm}$; spacing $1 \mathrm{~mm}$; image matrix $174 \mathrm{X}$ 349, 2 NEX; Field of Vision (FOV) 33 X 30cm; b-values were 50,400 and $800 \mathrm{~s} / \mathrm{mm}^{2}$. DWI was always performed before contrast enhancement.

C- Dynamic contrast MRI was done by 2dimensional fast spoiled gradient-recalled echo with fat suppression at T I WI (TR 4.3ms, TE $1.3 \mathrm{~ms}$ ), flip angle $80^{\circ}$ axial scan, FOV $34 \mathrm{X}$ $34 \mathrm{~cm}$, image matrix $448 \times 322,1 \mathrm{NEX}$, slice thickness $1 \mathrm{~mm}$, spacing $1 \mathrm{~mm}$ ). Prior to the imaging, a $12 \mathrm{G}$ venous lodging cannula was inserted. $\mathrm{T} 1$-weighted images were done in transverse plane prior to, and just after a bolus injection of $0.2 \mathrm{mmol}$ gadopentetate dimeglumine/kilogram of body weight at a rate of $3 \mathrm{ml} / \mathrm{s}$, followed by a $10-\mathrm{ml}$ saline flush using automatic injector (Medrad Spectris Solaris EP). 5-phase dynamic images were obtained at 1, 2, 3, 4 and 5 minutes respectively. Dynamic analysis with generation of enhancement percentage versus time curves done by positioning of Region of Interest (ROI) at center of identified enhancing lesions that had a diameter $>5 \mathrm{~mm}$ and masslike morphology according to Breast ImagingReporting and Data System. ADC value measurement: ROI was drawn in the center of the lesion at b-800 DWI. The location of the lesion was identified at the b-400 or b-50 image if the lesion was not visible at b-800 images. A small ROI was used at area close to tumor edge to obtain the greatest accuracy.

Post-processing of images includes image subtraction which was obtained by subtracting every pre-contrast images from its post-contrast series images, then time to Signal Intensity (S.I.) curve for any suspicious enhancing lesions and Maximum Intensity Projection (MIP) views were obtained. 
Image analysis: Each breast lesion was evaluated for site, size, signal intensity, its borders and fat necrosis, enhancing or not, pattern of enhancement either marginal or diffuse, if lesion showed mass like enhancement or non-mass like enhancement. The initial enhancement phase (within the first 2 minutes after contrast medium injection or until the curve started to change) was either slow or rapid. The delayed phase was either persistent, plateau, or washout patterns of enhancement. The most suspicious lesions were that which displayed rapid or medium initial enhancement followed by plateau or washout pattern on delayed phase. Visual analysis of S.I. on DWI and ADC maps was done before ADC value measurement. If the S.I. was high in DWI and low in ADC map, it has been considered as restricted diffusion. Moderate S.I. at both diffusion and ADC map was considered non-restricted. We considered lesions with MRI BI-RADS $\geq 4$ positives for malignancy and lesions with BI-RADS $\leq 3$ negative for malignancy.

III- Pathological assessment: All the patients had pathology report of primary breast cancer and also pathological assessment of newly developed lesions was obtained.

\section{IV- Statistical analysis:}

Data were statistically described in terms of range, mean \pm Standard Deviation $( \pm S D)$. All statistical calculations were done using computer program SPSS (Statistical Package for the Social Science). Student's $t$-test, Receiver-Operating Characteristic (ROC) curve was used to determine cutoff points, sensitivity and specificity for quantitative variables of interest and 2 X 2 tables used for calculation of false positive cases and false negative cases and diagnostic accuracy. $p$-value of $\leq 0.001$ was considered statistically highly significant while $p$-value of $<0.05$ was considered statistically significant.

\section{Results}

This prospective study was carried out on 30 female patients managed with BCT and had suspicious breast lesions at operative bed on follow-up scan by sono-mammography. The suspected breast lesions were examined by breast MRI. Age range was between 32.0-79.0Ys. With a mean of $49.13 \pm$ 12.42. All patients $(100 \%)$ underwent breast conservative surgery and received chemotherapy while 20 patients $(66.7 \%)$ received radiotherapy. According to histo-pathological finding of primary carcinoma in our study the invasive ductal carcinoma was the most common type found in 12 patients
(40\%) followed by invasive lobular carcinoma in 10 patients $(33.3 \%)$ then mixed ductal and lobular carcinoma in 8 patients $(26.7 \%)$.

As regarding morphologic MRI finding, 12 lesions (40\%) were regular shaped, 17 lesions $(56.7 \%)$ were irregular shaped and 1 lesion $(3.3 \%)$ showed non mass like pattern. 12 lesions (40\%) showed well-defined margin and 18 lesions (60\%) showed ill-defined margin.

On post contrast breast MRI, we detected 23 enhanced lesions (76.7\%) and 7 non enhanced lesions (23.3\%). Out of 23 enhanced lesions, 11 lesions $(36.7 \%)$ showed rim enhancement, 9 lesions $(30 \%)$ showed heterogeneous enhancement and 3 lesions (10\%) showed homogenous enhancement.

Type I curve Figs. $(1,2)$ in 13 lesions $(43.3 \%)$ showed persistent enhancement, enhancement continues over the entire dynamic series, the emerging curve is a more or less straight line denoting benign nature. Type II curve Fig. (3) 4 lesions (13.3\%) elicited plateau curve; enhancement reaches a steady state after the initial phase denoting suspicious nature. Type III curve Fig. (4) 6 lesions (20\%) had washout curve, enhancement decreases again directly after the strong signal increase in the early phase denoting malignant nature (Table 1).

On visual analysis of S.I. at diffusion sequence, 9 cases $(30 \%)$ showed diffusion restriction with ADC values in the range of 0.72-1.09 X $10^{-3} \mathrm{~mm}^{2} / \mathrm{s}$ with a mean of $0.88 \pm 0.13$ while 21 cases $(70 \%)$ showed no restricted diffusion with ADC values in the range of $1.22-1.81 \times 10^{-3} \mathrm{~mm} / \mathrm{s}$ with a mean of $1.38 \pm .18$ (Table 2).

According to the ADC values; 8 lesions (26.7\%) were loco-regional tumor recurrence Fig. (4), showed mean ADC values of $0.89 \pm 0.14 X$ $10^{-3} \mathrm{~mm} / \mathrm{s}$ and ADC range of (0.72-1.09 X 10 $\left.\mathrm{mm}^{2} / \mathrm{s}\right), 13$ lesions $(43.3 \%)$ were fat necrosis Fig. (2) showed mean ADC values of $1.33 \pm 0.15 \mathrm{X}$ $10^{-3} \mathrm{~mm} / \mathrm{s}$ and ADC range of (1.01-1.62 X 10 $\mathrm{mm}^{2} / \mathrm{s}$ ) and 9 lesions (30\%) were post-operative scar fibrosis Fig. (1) showed mean ADC values of $1.44 \pm 0.20 \times 10_{2}^{-3} \mathrm{~mm} / \mathrm{s}$ and ADC range of (1.22$1.81 \times 10^{-3} \mathrm{~mm}^{2} / \mathrm{s}$ ) (Table 3), Fig. (5).

The ROC curve Fig. (6) indicated a good statistical performance of the ADC values to predict the presence of malignant lesions (area under the curve $=0.991$ ).

According to BI-RADS classification depending on MRI study (including combined morphological, 
contrast enhancement pattern lesions), the breast lesions were divided into two groups:

- Group I include (BI-RADS 2, 3) denoting MRI non-suspicious breast lesions.

- Group II include (BI-RADS 4, 5) denoting MRI suspicious breast lesions.

The overall MRI examination including DCEMRI and DWI-MRI revealed 21 non-suspicious lesions (70\%) and 9 suspicious lesions (30\%) (Table 4).

In our study 8 breast lesions proved as recurrent malignancy, 2 of them (6.7\%) showed type II curve
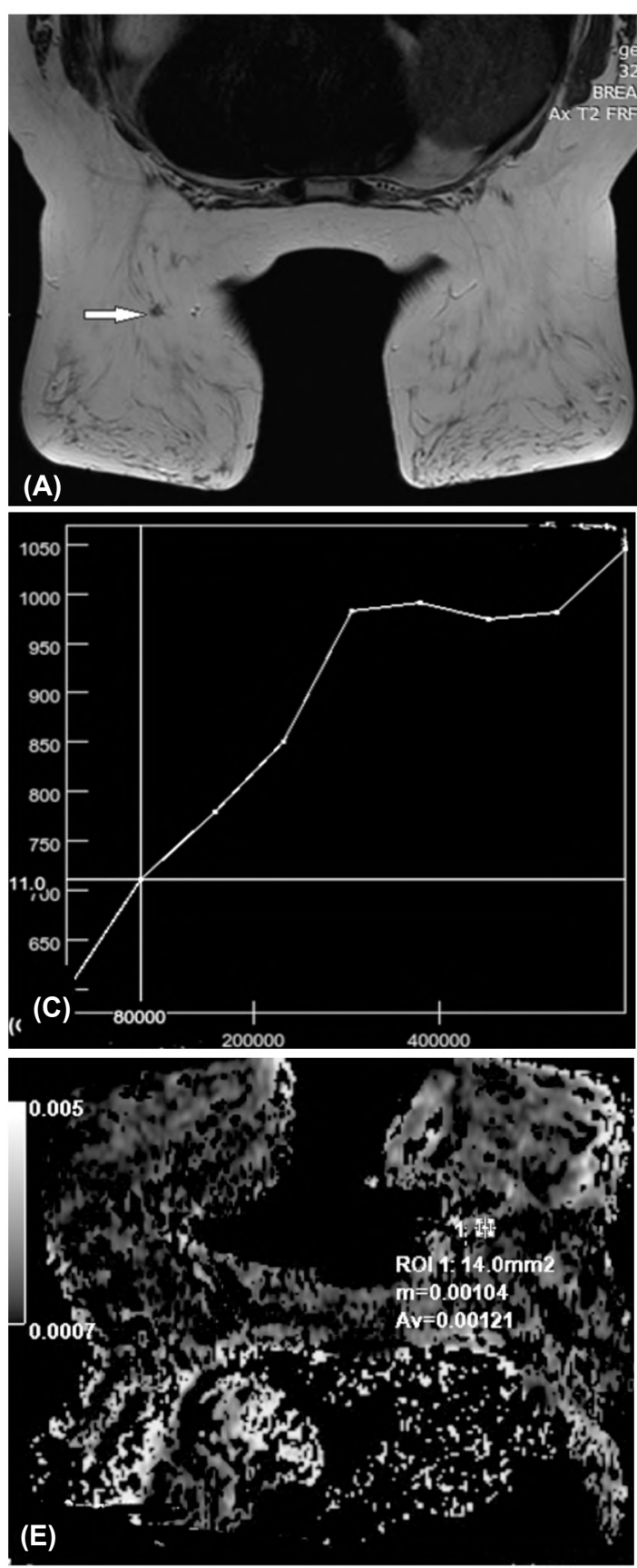

Fig. (3) and 6 lesions (20\%) showed type III curve Fig. (4) (Table 5).

DCE-MRI diagnosis (considering lesion morphology, enhancement pattern) overestimated 2 lesions were scored BIRADS 4 and proved pathologically as fat necrosis. DCE-MRI diagnosis correlated with histopathological diagnosis with $80 \%$ positive predictive value for recurrent lesions. (Table 6).

In our study MRI showed $100 \%$ sensitivity, 95.4\% specificity, $88.9 \%$ positive predictive value, $100 \%$ negative predictive value and $96,6 \%$ accuracy in evaluation of breast cancer patients after breast conservative therapy (Table 7).
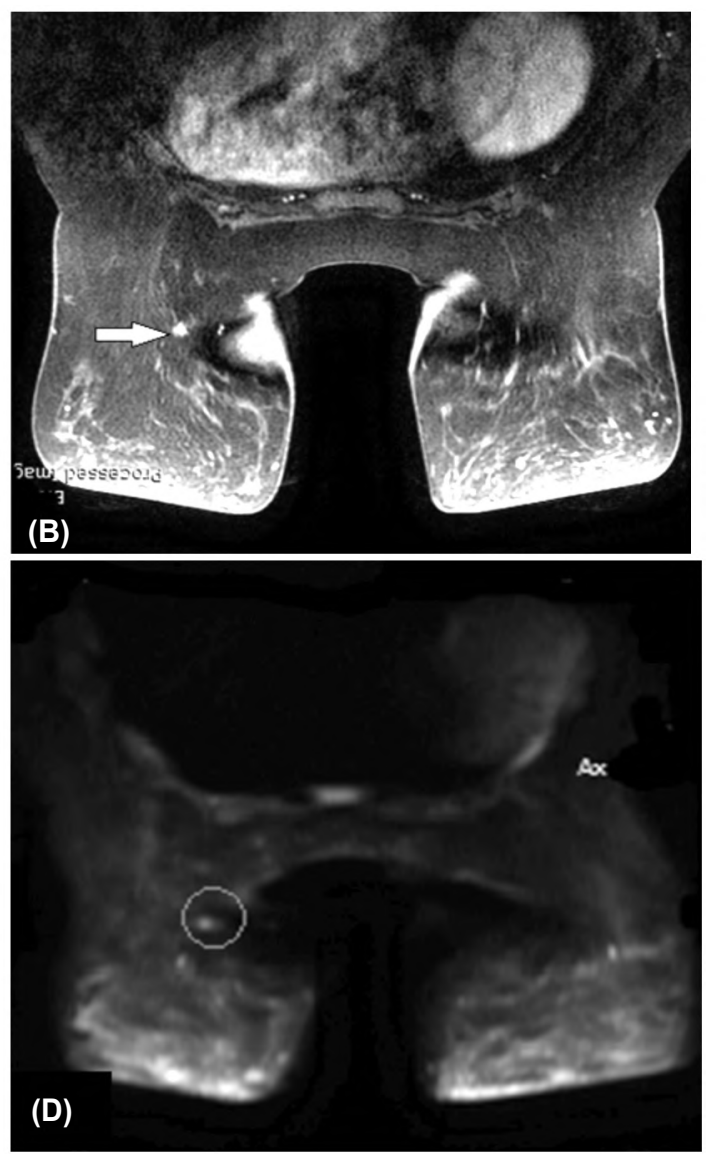

Fig. (1): A 56 year-old female patient had left breast cancer 3 years ago. Histopathology revealed invasive ductal carcinoma grade (II) with vascular invasion treated with conservative surgery followed by chemotherapy and radiotherapy and now on hormonal treatment. (A) Axial T2WI demonstrated irregular area of low S.I. at lower inner quadrant of Lt. breast. (B) Axial T 1 WI post contrast showed enhancement at area of interest. (C) Kinetic assessment of enhanced area showed rising curve denoting benign nature (D) DWI showed hyper intense signal. (E) ADC map showed hyper intense signal with ADC value $1.2 \times 10^{-} \mathrm{mm}$. MRI diagnosis was post-operative scar. Histopathology revealed no residual or recurrent tumor. 

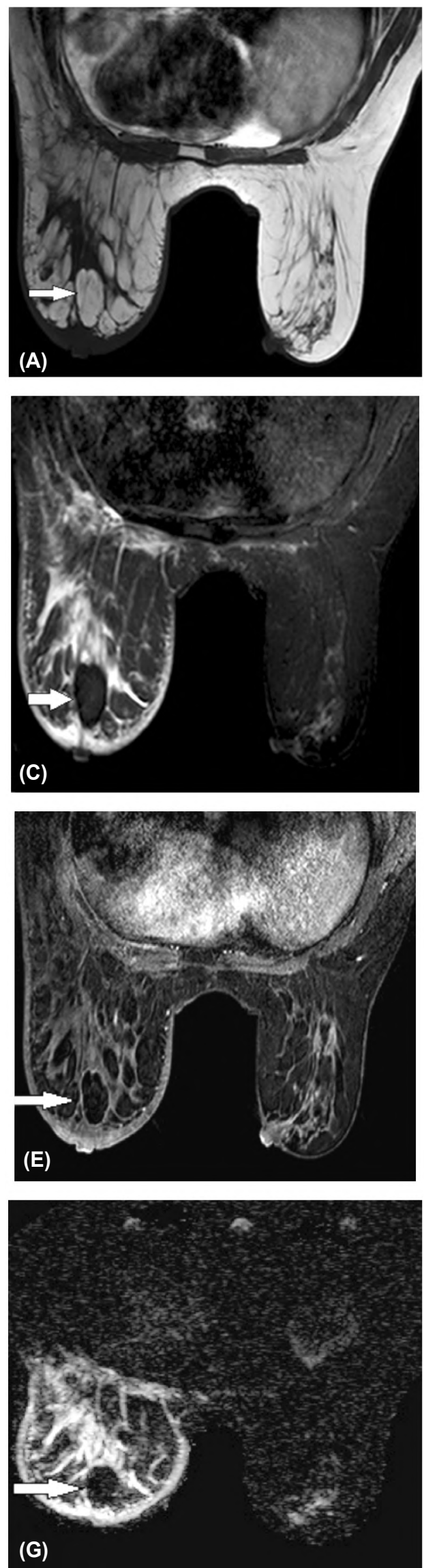
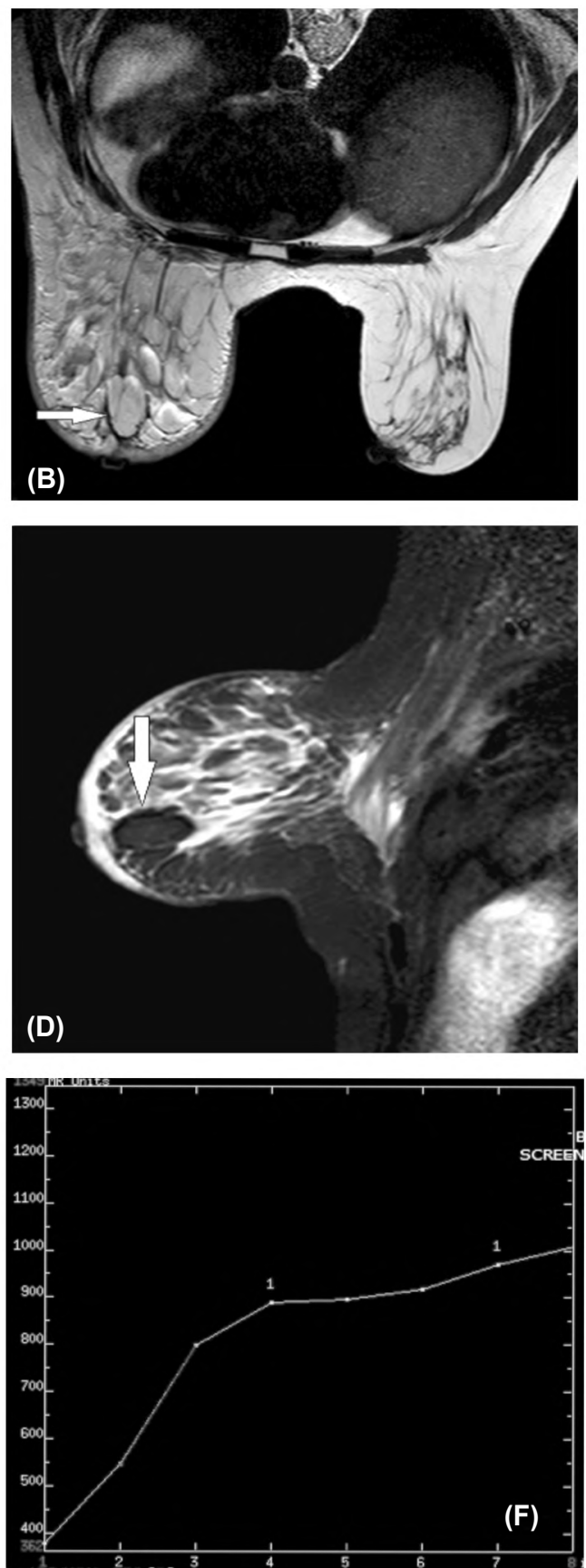

Fig. (2): A 54 year-old female patient had left breast cancer underwent left lumpectomy 2 years ago, histopathology revealed infiltrating lobular carcinoma (grade II), received chemotherapy and radiotherapy. After 19 months she was complaining of palpable mass in left breast at site of previous surgery. (A \& B) Axial T $1 \mathrm{WI}$ and T2WI showed well defined isointense lesion at upper outer quadrant of left breast. (C \& D) Axial and sagittal STIR demonstrated loss of signal. (E) Axial T 1 WI post contrast showed faint marginal enhancement. (F) Kinetic assessment showed type I rising curve (G) DWI showed a hypo intense signal with ADC value $1.42 \times 10^{-3} \mathrm{~mm}^{2} / \mathrm{s}$. Histopathology revealed fat necrosis. 

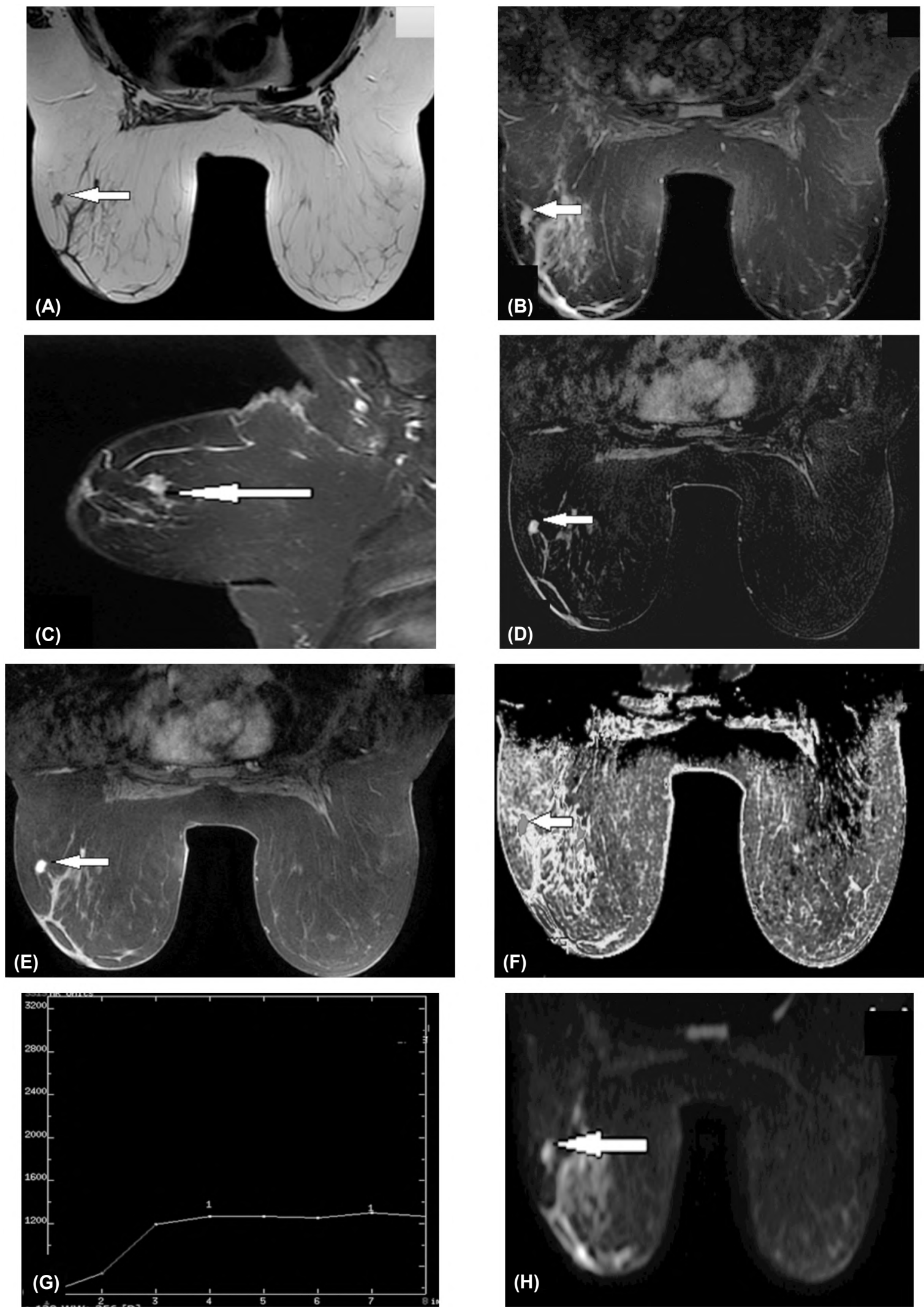

Fig. (3): A 52-year-old female patient had left breast cancer, underwent conservative surgery 4 years ago, histopathology revealed invasive ductal carcinoma (grade II) received chemotherapy and radiotherapy and now on hormonal treatment. She presented for routine follow-up. (A) Axial T2WI showed small lobulated hypointense mass at upper outer quadrant of left breast. (B,C) Axial and sagittal STIR demonstrate no signal loss. (D) Subtraction image (E) Axial T 1 WI post contrast showed homogeneous mass enhancement. (F) Color coding map showed neovascularization of the lesion (G) Kinetic assessment showed type II plateau curve denoting suspicious nature (H) DWI showed hyper intense signal (BIRADS 4) and ADC value $0.75 \mathrm{X}$ $10 \mathrm{~mm} / \mathrm{s}$. MRI diagnosis was neoplastic recurrence. Histopathology confirm recurrence. 

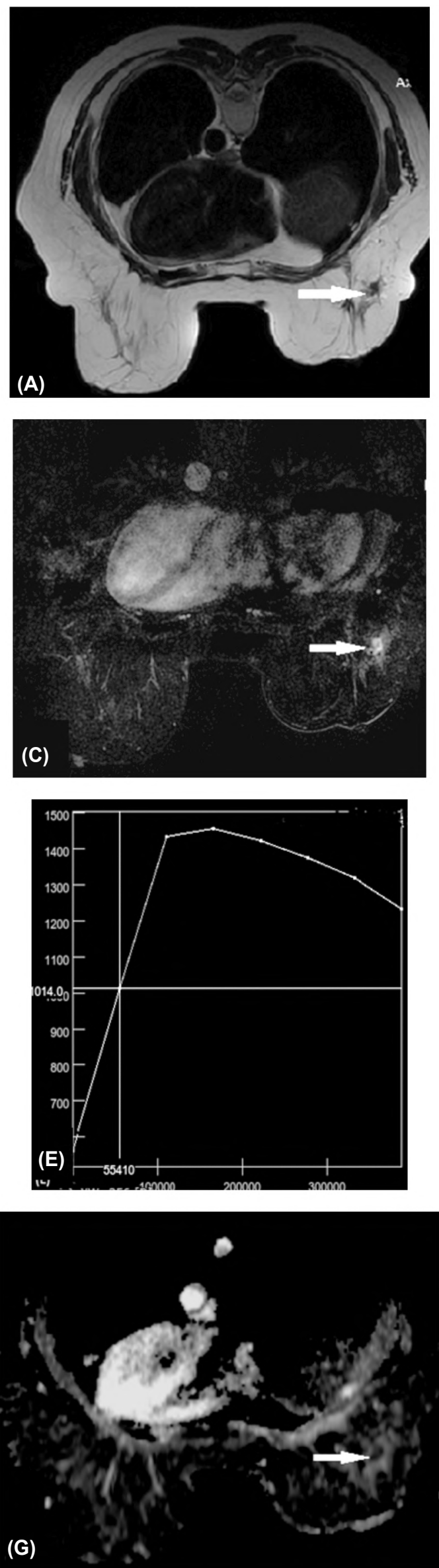
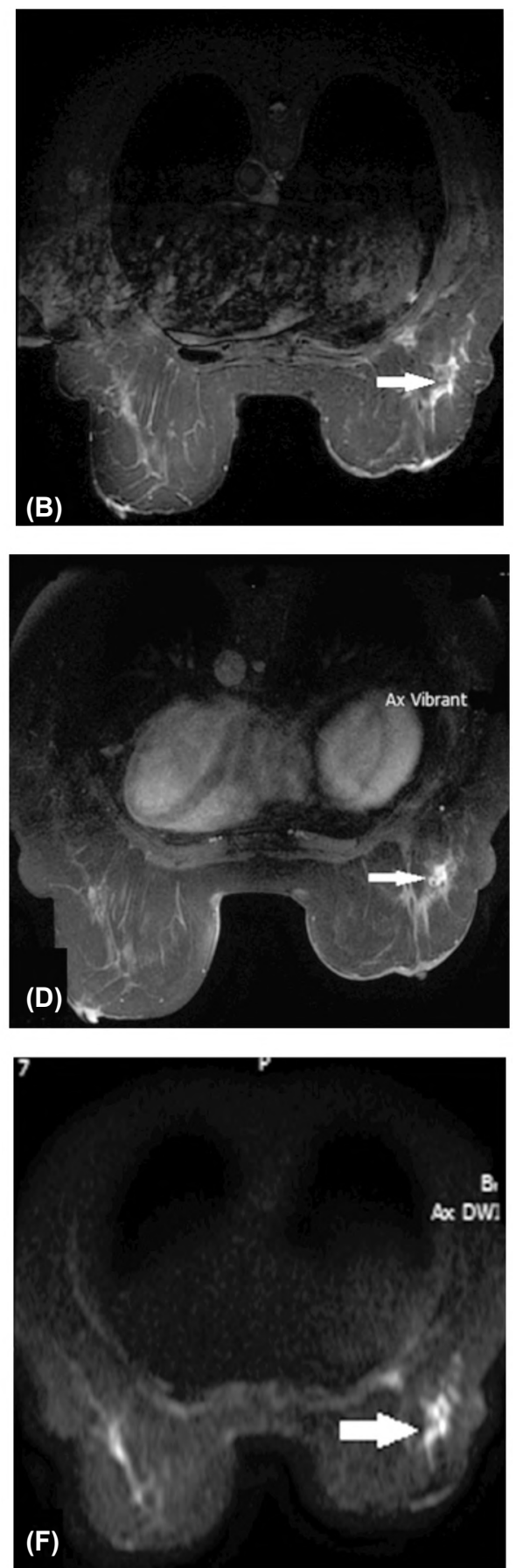

Fig. (4): A 55-year-old female patient, had right breast cancer underwent right lumpectomy 3 years ago, histopathology revealed invasive ductal carcinoma (grade II), received chemotherapy and radiotherapy. (A) Axial T2WI \& (B) Axial STIR demonstrated speculated mass at upper outer quadrant of right breast. (C) Subtraction image \& (D) Axial $\mathrm{T}_{1} \mathrm{WI}$ post contrast showed heterogeneous mass enhancement. (E) Kinetic assessment of enhanced lesion showed washout curve denoting malignant nature (F) DWI showed hyper intense signal (arrow) denoting restricted diffusion (BIRADS 5). (G) ADC map showed hypo intense lesion (arrow) with ADC value $1.01 \times 10 \mathrm{~mm} / \mathrm{s}$. MRI diagnosis was neoplastic recurrence. Histopathology revealed recurrent IDC. 


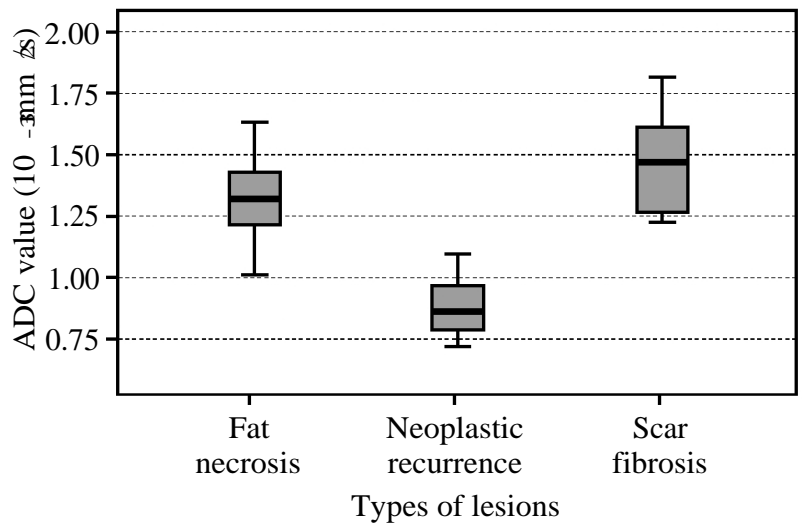

Fig. (5): Box plots graphs of Apparent Diffusion Coefficient (ADC) values for loco-regional neoplastic recurrence and benign post-operative lesions.

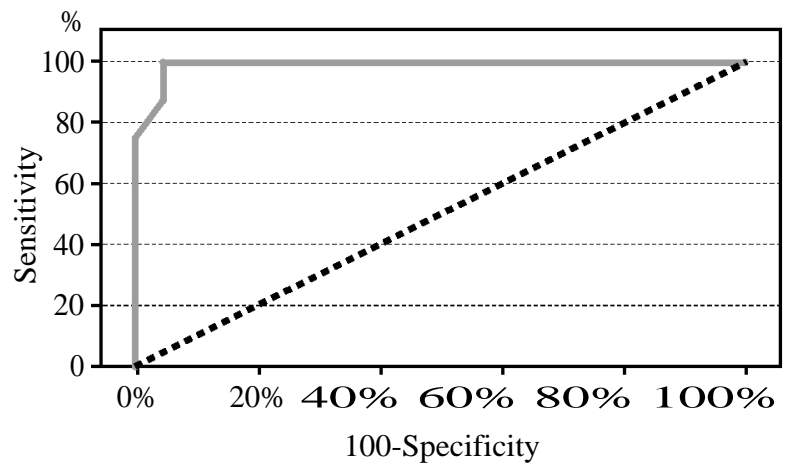

Fig. (6): Receiver Operating Characteristic (ROC) curve of ADC value in the studied group.

Table (1): Types of dynamic curves at enhanced 23 breast lesions.

\begin{tabular}{lccl}
\hline Types of dynamic curves & No. & $\%$ & Interpretation \\
\hline Type I & 13 & 43.3 & Benign lesions \\
Type II & 4 & 13.3 & Suspicious lesions \\
Type III & 6 & 20 & Malignant lesions \\
\hline Total & 23 & 76.6 & \\
\hline
\end{tabular}

Table (2): DWI \& ADC values in our 30 patients.

\begin{tabular}{lcc}
\hline & \multicolumn{2}{c}{ MRI diffusion finding } \\
\cline { 2 - 3 } & $\begin{array}{c}\text { No diffusion restriction } \\
\mathrm{n}=21(70 \%)\end{array}$ & $\begin{array}{c}\text { Restricted diffusion } \\
\mathrm{n}=9(30 \%)\end{array}$ \\
\hline$A D C:$ & & \\
Range & $1.22-1.81$ & $0.72-1.09$ \\
Mean $\pm \mathrm{SD}$ & $1.38 \pm 0.18$ & $0.88 \pm 0.13$ \\
\hline
\end{tabular}

Table (3): Different ADC values for recurrent tumor and benign post-operative breast lesions $(n=30)$.

\begin{tabular}{|c|c|c|c|}
\hline & \multirow{2}{*}{$\begin{array}{l}\text { No. of } \\
\text { lesions }\end{array}$} & \multicolumn{2}{|c|}{ ADC values $\left(10^{-3} \mathrm{~mm}^{2} / \mathrm{s}\right)$} \\
\hline & & Range & Mean \pm SD \\
\hline \multicolumn{4}{|l|}{ Types of lesions: } \\
\hline Local recurrence & $8 \quad(26.7 \%)$ & $0.72-1.09$ & $0.89 \pm 0.14$ \\
\hline Fat necrosis & $13(43.3 \%)$ & $1.01-1.62$ & $1.33 \pm 0.15$ \\
\hline Scar fibrosis & $9 \quad(30 \%)$ & $1.22-1.81$ & $1.44 \pm 0.20$ \\
\hline
\end{tabular}

Table (4): The BIRADS classification depending on MRI findings of the studied patients $(\mathrm{N}=30)$.

\begin{tabular}{lccccc}
\hline & \multicolumn{3}{c}{ Final MRI finding } \\
\cline { 2 - 3 } & \multicolumn{2}{c}{$\begin{array}{c}\text { Suspicious } \\
\text { (BI-RADS 4,5) }\end{array}$} & & \multicolumn{2}{c}{$\begin{array}{c}\text { Non-suspicious } \\
\text { (BI-RADS 2,3) }\end{array}$} \\
\cline { 2 - 3 } \cline { 5 - 6 } & No. & $\%$ & & No. & $\%$ \\
\hline DCE-MRI & 10 & 33.3 & & 20 & 66.7 \\
SWI-MRI & 9 & 30 & & 21 & 70 \\
\hline
\end{tabular}

Table (5): Correlation between dynamic curve type and recurrent lesions $(n=8)$.

\begin{tabular}{ccc}
\hline & No. & $\%$ \\
\hline Type of curve: & & \\
Type II curve & 2 & 6.7 \\
Type III curve & 6 & 20 \\
\hline
\end{tabular}

Table (6): Correlation between the DCE-MRI finding and local recurrence \& other post-operative changes.

\begin{tabular}{|c|c|c|c|c|}
\hline & \multicolumn{4}{|c|}{ DCE-MRI diagnosis } \\
\hline & \multicolumn{2}{|c|}{$\begin{array}{l}\text { Suspicious } \\
\quad(\mathrm{n}=10)\end{array}$} & \multicolumn{2}{|c|}{$\begin{array}{c}\text { Non- } \\
\text { suspicious } \\
(\mathrm{n}=20)\end{array}$} \\
\hline & No. & $\%$ & No. & $\%$ \\
\hline \multicolumn{5}{|l|}{ Histopathological result: } \\
\hline Recurrence $(n=8)$ & 8 & 80 & 0 & 0.0 \\
\hline Post-operative changes $(n=22)$ & 2 & 20 & 20 & 100 \\
\hline
\end{tabular}

Table (7): MRI sensitivity, specificity, positive predictive value, negative predictive value and accuracy.

\begin{tabular}{lcccc}
\hline Sensitivity & Specificity & PPV & NPV & Accuracy \\
\hline $100.0 \%$ & $95.4 \%$ & $88.9 \%$ & $100.0 \%$ & $96.6 \%$ \\
\hline
\end{tabular}

\section{Discussion}

The current study included 30 female patients with suspicious breast lesions after BCS. All patients were referred to do MRI breast to verify nature of these suspicious lesions.

In our study we found suspicious morphologic criteria as irregular, speculated outline and illdefined margin in 18 lesions (60\%) while in 12 lesions $(40 \%)$ had benign morphologic criteria as well defined margin and regular outline. Our results agreed with that announced by Nunes et al., 2001 [12] who noted that the association of speculated and irregular border of focal mass with malignancy. Lobulated and smooth masses had a negative predictive value for malignancy.

In our study breast MRI kinetic analysis curves showed: Type I rising curve detected in 13 lesions 
(43.3\%) considered as benign lesions. Type II plateau curve detected in 4 lesions $(13.3 \%)$ considered as benign lesions (but on DWIMRI 2 of them was recurrent malignancy and the other 2 cases was post-operative scar). Type III washout curve detected in 6 lesions (20\%) considered as malignant lesions. Type III curve was the most common type in the pathologically proved malignant cases as it was found in 6 out of 8 total malignant lesions. This is in agree with the study done by Jack, 2011 [13], who found that type III (washout) curve was $86.49 \%$ (32/37). We also agreed with Kuhl, 2000. [14], as he reported that more malignant cases with type III curve than type II curve.

In the current study there were 2 lesions $(6.7 \%)$ pathologically proved as fat necrosis overestimated by MRI BIRADS to be IV, one of them showed washout curve and the other showed plateau curve. This is in agreement with Drukteinis, 2012 [11] who stated that fat necrosis is a common and challenging pitfall in interpretation of post-BCT on MR imaging.

In our study, out of 9 lesions (30\%) were proved pathologically as post-operative scar fibrosis in 6 of them with their MRI showed an area of architectural distortion and no enhancement was observed on dynamic post-contrast study while the remaining 3 lesions (10\%) showed irregular margin, heterogeneous enhancement and Type I time signal intensity curve. The conventional MRI morphologic appearance of focal recurrent mass was similar to scar fibrosis. The lack of post contrast enhancement was the main differentiating point in this study as well as other studies in the literature [15].

On DWI-MRI in this study, all cases of postoperative scarring showed high ADC values than loco-regional tumor recurrence so ADC values can be used to distinguish loco-regional tumor recurrence from benign breast changes after BCT. All cases of loco-regional tumor recurrence showed high signal intensity on DWI and low signal intensity on ADC map with mean ADC values of $0.89 \pm$ $0.14 \times 10_{2}^{-3} \mathrm{~mm} / \mathrm{s}$ and ADC range of $(0.72-1.09$ $\mathrm{X} 10^{-3} \mathrm{~mm} / \mathrm{s}$ ) which is in accordance with recent study done by Sahin et al., 2013 [16].

The mean ADC values of post-operative scars in our study measures $1.44 \pm 0.20 \times 10_{-3}^{-3} \mathrm{~mm}^{2} / \mathrm{s}$ with ADC range of about 1.22-1.81 X $10^{-3} \mathrm{~mm} / \mathrm{s}$. Multiple studies $[\mathbf{1 7 , 1 8 ]}$ stated that post-operative granulation tissue had a high ADC value $(2.66 \mathrm{X}$ $10^{-3} \mathrm{~mm} / \mathrm{s}$ ) which in agreement with our study. Recent meta-analysis has determined that an ADC value $>1.2 \times 10^{-3} \mathrm{~mm}^{2} / \mathrm{sec}$ speaks for benignancy [17] and other recent study [19] stated that the average ADC for scar tissue was $1.89 \mathrm{x}_{3} 10^{-3} \mathrm{~mm} / \mathrm{s}$ and ADC range was (1.43-2.20 X 10 $\left.{ }^{-3}\right)$ which are in accordance with our results.

In our cases of fat necrosis, showed mean ADC values of $1.33 \pm 0.15 \times 10^{-3} \mathrm{~mm} / \mathrm{s}$ and $A D C$ range of (1.01-1.62 $\left.\left.12 \times 10^{-3} \mathrm{~mm}^{2} / \mathrm{s}\right) \times 10^{-3} \mathrm{~mm}^{2} / \mathrm{s}\right)$ which in agreement with recent study [21]

One exception in our study is one case of fat necrosis showed low ADC value (1.01 X 10 $\mathrm{mm}^{2} / \mathrm{s}$ ) mimicking recurrence and this in agreement with previous study reported that fat necrosis and scar tissue can show lower ADC values [20,21] Fortunately, this case was diagnosed by detection of fat signal in the conventional sequences; presence of high signal in the lesion in $\mathrm{T}_{1} \mathrm{WI}$ showed signal drop in T2 STIR.

We considered the cut off ADC value between benign and malignant breast pathologies as 1.09 $\mathrm{X} 10 \mathrm{~mm} / \mathrm{s}$ and this matched with further study [22].

When adding the data elicited by the DWI to the DCE-MRI assessment, the statistical analysis presented $100 \%$ sensitivity, $95.4 \%$ specificity, 88.9\% PPV, $100 \%$ NPV and $96.6 \%$ accuracy. And this matched well with previous study [23] reported that the sensitivity, and specificity for MRI in the differentiating loco-regional tumor recurrence from benign breast lesions were $100 \%$ and $94.7 \%$, respectively.

\section{In Conclusion:}

Combination of functional \& dynamic MRI techniques in follow-up after BCT could help to avoid biopsy in positive patients and to send negative patients to follow-up, reserving biopsy in the cases with a clinico-radiological mismatch. Breast MRI should be the imaging modality of choice in detection of the tumoral recurrence, and differentiating it from other post-operative complications.

Limitaion of the study: This tudy was only conducted on little number of patients (30 patients).

\section{References}

1- SAIF EL-NASR S., ABDEL RAHMAN R., et al.: Role of diffusion weighted imaging and dynamic contrast enhanced MR mammography to detect recurrence in breast cancer patients after surgery, Egypt J. Radiol. Nucl. Med., 47: pp. 1151-7, 2016.

2- FISHER B., ANDERSON S. and REDMON C.K.: Reanalysis and results after 12 years of follow-up in a randomized clinical trial comparing total mastectomy with 
lumpectomy with or without irradiation in the treatment of breast cancer, N. Engl. J. Med., 333: pp. 1456-61, 1995.

3- RINALDI P., GIULIANI M., BELLI P., et al.: DWI in breast MRI: Role of ADC value to determine diagnosis between recurrent tumor and surgical scar in operated patients. Eur. J. Radiol., 75 (2): pp. e114-e123. 2010.

4- KURTZ J.M., AMALRIC R., BRANDONE H., et al.: Local recurrence after breast conservation surgery and radiotherapy: Frequency, time course and prognosis Cancer, 63: pp. 112-1917, 1989.

5- RECHT A., SILEN W., SCHNITT S.J., et al.: Time course of local recurrence following conservative surgery and radiotherapy for early stage breast cancer Int. J. Radiat. Onclol. Biol. Phys., 15: pp. 255-61, 1988.

6- FISCHER U., KOPKA L. and GRABBE E.: Breast carcinoma: Effect of pre-operative contrast-enhanced MR imaging on the therapeutic approach Radiology, 213: pp. 881-8, 1999.

7- KOH D.M. and COLLINS D.J.: Diffusion-weighted MRI in the body: Applications and challenges in oncology, AJR Am. J. Roentgenol., 188 (6): pp. 1622-35, 2007.

8- WOODHAMS R., RAMADAN S., STANWELL P., et al.: Diffusion-weighted imaging of the breast: Principles and clinical applications, Radiographics, 31 (4): pp. 105984, 2011.

9- PETRALIA G., BONELLO L., PRIOLO F., et al.: Breast MR with special focus on DW-MRI and DCE-MRI, Cancer Imaging, 28 (11), pp. 76-90, 2011.

10- CHEN J.H., MEHTA R. S., BAEK H.M., et al.: Clinical characteristics, biomarkers of breast cancer associated with choline concentration measured by $1 \mathrm{H}$ MR spectroscopy N.M.R. Biomed., 24 (3): pp. 316-24, 2011.

11- DRUKTEINIS J., GOMBOS E., RAZA S., et al.: MR Imaging Assessment of the Breast after Breast Conservation Therapy: Distinguishing Benign from Malignant Lesions. RSNA, Volume 32, Issue 1, 2012.

12- NUNES L.W., SCHNALL M.D. and OREL S.G.: Update of breast MR imaging architectural interpretation model. Radiology, 219 (2): 484-94, 2001.

13- JACK P.: Magnetic Resonance Imaging Study for Breast Lesions. Medical Research, Retrieved from http://www res-medical.com/clinical-medicine/19800, 2011.
14- KUHL C.K. and SCHILD H.H.: Dynamic image interpretation of MRI of the breast. Journal of magnetic resonance imaging: JMRI, 12 (6): 965-74, 2000.

15- DAO T.H., RAHMOUNI A., CAMPANA F., et al.: Tumor recurrence versus fibrosis in the irradiated breast: Differentiation with dynamic gadolinium-enhanced MR imaging. Radiology, 187 (3): 751-5, 1993.

16- PARTRIDGE S.C., MULLINS C.D., KURLAND B.F., et al.: Apparent diffusion coefficient values for discriminating benign and malignant breast MRI lesions: Effects of lesion type and size. AJR American Journal of Roentgenology, 194 (6): 1664-73, 2010.

17- SAHIN C. and AR BAL E.: The role of apparent diffusion coefficient values in the differential diagnosis of breast lesions in diffusion-weighted MRI. Diagn. Interv. Radiol. 19: 457-62, 2013.

18- RANILDI P., GIULAINI M., BELLI B., et al.: DWI in breast MRI: Role of ADC value to determine diagnosis of tumor between tumor recurrence and surgical scar in operated patients. European Journal of Radiology, 75; 2 : e114-e 123, 2010.

19- TSUSHIMA Y., et al.: Magnetic resonance (MR) differential diagnosis of breast tumors using apparent diffusion coefficient (ADC) on 1.5 T. JMRI, 30: 249-55, 2009.

20- KELCZ F. and HAIN K.S.: Post Lumpectomy MRI: Using diffusion weighted imaging (DWI) to distinguish benign from malignant tissues. Mag. Reson. Med., 18: 4535, 2010 .

21- GONZALES M.A., CASTAÑON A.I., BALTAR B.N., et al.: Diffusion weighted MR Imaging assessment after breast conservative treatment. ECR, C-1065, 2012.

22- NILSEN L.B., FANGBERGET A., GEIER O., et al.: Quantitative analysis of Diffusion-weighted magnetic resonance imaging in malignantbreast lesions using different bvalue combinations. Eur. Radiol., 23 (4): 1027 33, 2013.

23- ABDULGHAFFARA W., TAG-ALDEINB M., et al.: Role of diffusion-weighted imaging and apparent diffusion coefficient in differentiating between local tumor recurrence and benign breast changes after breast conservative surgery Global J. Med. Res.: D Radiol, Diagn. Imag. Instrum., Version 1.0. 2014. 


\section{مراقبة آفات الثدى بعد علاج الثدى التحفظى: التئي

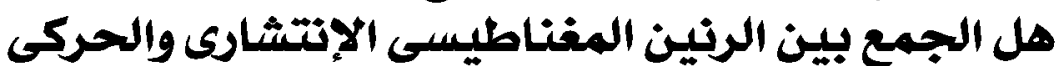

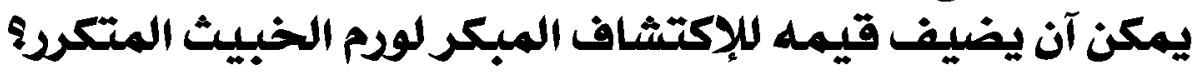

وتفريقه من الآفات الآخرى بتآثير الجمع الجراحة. الرنين المغناطيسى الإنتشارى والحركى للإكتشاف المبكر للودم الخبيث المتكرد بعد علاج الثَى التحفظى

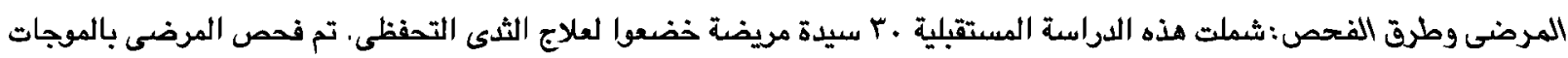

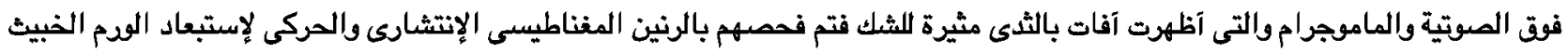

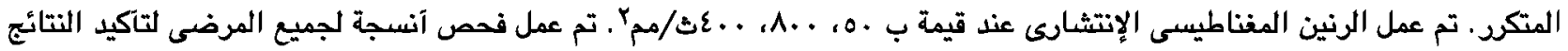
والتى آعتبرت المقياس الذهبى لهذه الدراسة.

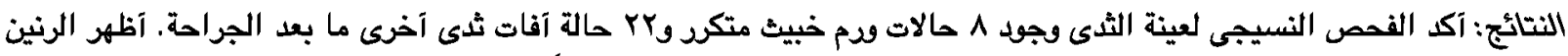

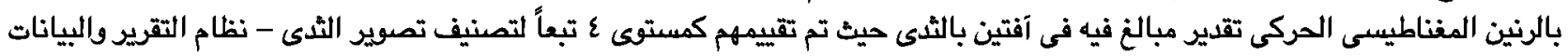

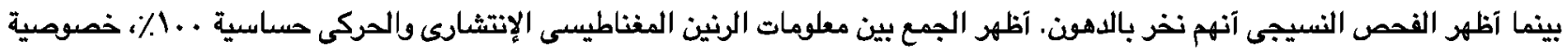

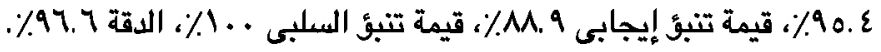

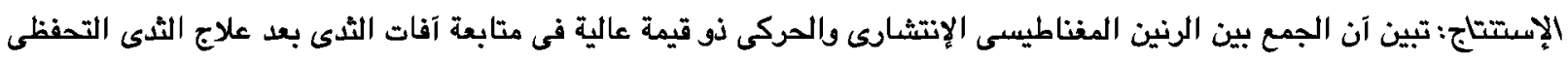

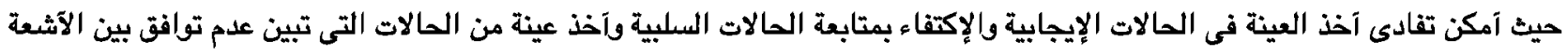

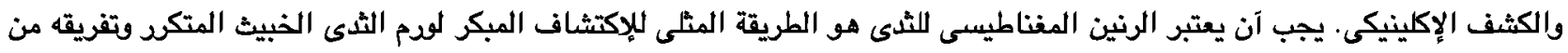

